\title{
The disappearing string of pearls: Super Star Clusters in a nuclear star-forming ring of NGC 2328
}

\author{
Sudhanshu Barway ${ }^{* \dagger}$ \\ South African Astronomical Observatory, P.O. Box 9 Observatory, Cape Town, South Africa \\ E-mail: barwayesaao.ac.za
}

Petri Vaisanen

South African Astronomical Observatory, P.O. Box 9 Observatory, Cape Town, South Africa

E-mail: petriesaao.ac.za

\section{Zara Randriamanakoto}

South African Astronomical Observatory, P.O. Box 9 Observatory, Cape Town, South Africa

E-mail: zara@saao.ac.za

\begin{abstract}
An analysis of the super star cluster population in a low-luminosity early type galaxy, NGC 2328, is discussed. The clusters are found in a tight star-forming nuclear spiral/ring pattern. We also identify a bar from structural 2D decomposition. These massive clusters are forming very efficiently in the circum-nuclear environment, they are young, possibly all less than 30 Myr of age. The clusters show an azimuthal age gradient, consistent with a "pearls-on-a-string" formation scenario suggesting bar driven gas inflow. The cluster mass function has a robust down-turn at low masses at all age bins. Assuming clusters are born with a power-law distribution, this indicates extremely rapid disruption at time- scales of just several Myr. If found to be typical, it means that clusters born in dense circum-nuclear rings do not survive to become old globular clusters in noninteracting systems.
\end{abstract}

SALT Science Conference 2015 -SSC2015-

1-5 June, 2015

Stellenbosch Institute of Advanced Study, South Africa

\footnotetext{
${ }^{*}$ Speaker.

${ }^{\dagger}$ Based on article published in Astrophysical Journal Letters, 2014, 797, 16. I acknowledge financial support from the NRF research grant (UID-93727).
} 


\section{Introduction}

Gas reservoirs in early-type galaxies (ETGs) have turned out to be fairly common and they can exhibit on-going star formation (SF). The origin of the gas and cause for SF are still under discussion however. In principle the systems can be governed by internal effects, or by external galaxy interaction/merging related effects. What makes NGC 2328 worthy of a further investigation is its morphologically well defined ISM characteristics due to presence of young massive star clusters (YMC). These YMCs, also known as super star clusters (SSC), have been found to be ubiquitous in interacting gas rich galaxies and dwarf starbursts [1]. There are not many studies of YMCs in ETGs, though when they are found they tend to inhabit nuclear star-forming regions [2]. YMCs thus appear to form in high-pressure environments - but they may also dissolve quickly there [3]. Which clusters survive to perhaps become old globular clusters, and in which conditions?

A study of the YMCs in NGC 2328, a little-studied low-luminosity $\left(\mathrm{M}_{V}^{t o t}=-18.5 \mathrm{mag}\right)$ galaxy at (the adopted) $18 \mathrm{Mpc}$ distance with an S0 classification is presented in this paper. In the centre of the largely featureless isolated galaxy, HST images reveal a tight and bright nuclear spiral, or a ring, of $\sim 200 \mathrm{pc}$ radius. The feature resolves itself into numerous "hot spots", point sources which are candidate star clusters. YMCs, typically $<5 \mathrm{pc}$ in size, would not be resolved at the distance of the galaxy, where the resolution of the images we use corresponds to $\sim 9 \mathrm{pc}$. Other studies have found both young and intermediate age clusters in star-forming rings [2, 4], while recently [5] demonstrated very rapid disruption in a starburst ring of a spiral galaxy. The aim of this study is to define the age and mass distribution of YMCs in NGC 2328 to explore the effect of the ring-environment on star clusters.

\section{Observation and data reduction}

\subsection{Optical and NIR HST data}

NGC 2328 was observed using the WFPC2 and newly installed WFC3 on board the HST. All data used in this paper were downloaded from The Hubble Legacy Archive (HLA) ${ }^{1}$ at the Space Telescope Science Institute (STScI). WFPC2 images were obtained with the F555W and F814W filters (henceforth referred to as the $V$ and $I$ bands, respectively) as a part of HST program 5999 (PI: A. C. Phillips). For this study, we only used the PC chip which has a 0.05 arcsec pixel scale, to study the central region of NGC 2328. We also used the WFPC2 image in the F658N filter sampling emissions from $\mathrm{H} \alpha+[\mathrm{NII}]$ region (the $\mathrm{H} \alpha$ image henceforth). A NIR image in the F160W filter (we will refer to it as the H-band) was obtained using WFC3 as a part of HST program 11219 (PI: A. Capetti). The images were aligned such that North is up and East is right, the WCS system was fine-tuned using the galaxy centre and two GSC-2 stars in the field, and the NIR image was resampled to a common pixel scale of 0.05 arcsec with the the optical data, so that images in all bands were exactly aligned. Figure 1 shows a pseudo-colour (RGB) image of NGC 2328 created using the WPC $3 H$ band as red and WFPC $2 I$ and $V$ frames as green, and blue channels, respectively. We note the central region of NGC 2328 appears to be dominated by a tight spiral pattern, or a ring, with lots of bright star forming knots, and with obvious dust patches. An accurate estimation of the PSF is crucial for determination of the faint nuclear structure, surface photometry

\footnotetext{
${ }^{1}$ http://hla.stsci.edu/hlaview.html
} 
and 2D image bulge/disk decomposition analysis. Stars in the field would be the best source of a PSF having gone through the same optics and reduction as the target galaxy. However, in our work we used the PC chip images in the optical and there are no suitable PSF stars due to the small field-of-view. An alternative is to create a simulated PSF and we thus generated model PSFs using the Tiny Tim software [6] for the $V, I$ and $H$ bands at location of the galaxy centre on the chip.

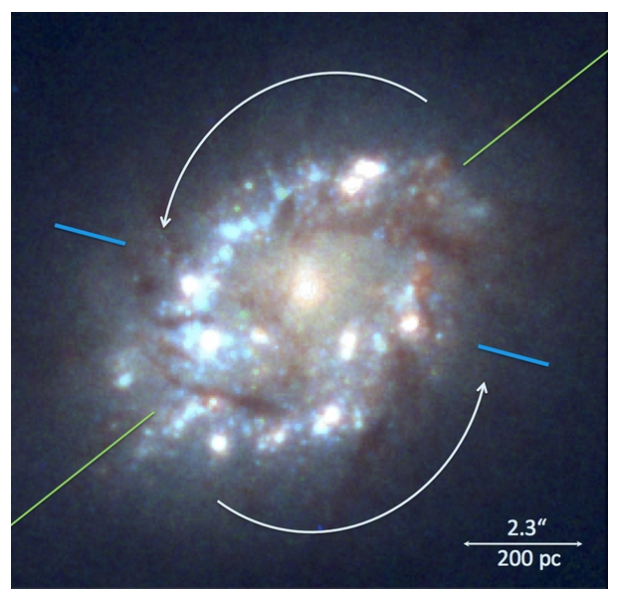

Figure 1: Three-color $V, I$, and $H$ HST view of NGC 2328; north is up, east is left. The young star clusters in the tight star forming ring (or spiral) pattern have an azimuthal age gradient. YMCs get younger along the direction of the arrows with the short markers indicating the approximate location of the youngest populations, while the longer markers show the P.A. of a bar.

\subsection{SALT long-slit spectroscopy}

NGC 2328 was observed with the Robert Stobie Spectrograph in long-slit mode at the Southern African Large Telecope in Sutherland, South Africa on May 15/16, 2011. The 0.6 arcsec slit used was positioned at $\mathrm{PA}=120$ through the centre of the galaxy. We used the PG0900 grating providing $2.29 \AA$ ( $0.98 \AA$ per binned pixel) resolution over the $3630-6730 \AA$ range. Two exposures of $600 \mathrm{sec}$ were taken, along with an Argon arc lamp for wavelength calibration as well as calibration system flat fields. The on-chip $2 \times 2$ binning gives a spatial scale of $0.253 \operatorname{arcsec~pix}^{-1}$, while the seeing during the science observation was $\sim 1.6$ arcsec. The SALT data pipeline PySALT products were used for primary reductions including the overscan, gain, cross-talk corrections, and mosaicing. Further routines in IRAF ${ }^{2}$ twodspec package and MIDAS ${ }^{3}$ were used for wavelength calibration (resulting in a $\sigma=0.15 \AA$ ), $2 \mathrm{D}$ frame transformation, background subtraction and tracing. The relative spectral shape was calibrated using a spectrophotometric standard star, LTT 4364, observed during the same night with the same setup, and velocities were corrected for heliocentric motion.

\footnotetext{
${ }^{2}$ IRAF is distributed by the NOAO, which is operated by the AURA under cooperative agreement with the NSF.

${ }^{3}$ Munich Image Data Analysis System is distributed by ESO.
} 


\section{Analysis}

\subsection{Galaxy Structure}

NGC 2328 is classified as S0 in the RC3 catalog [7] but (R)SBA0 in NED ${ }^{4}$. We derived structural parameters from the WFC3 H-band image using GALFIT [8]. A combination of Sersic, Exponential and Gaussian functions gives the best-fit model. The results indicate that NGC 2328 has no bulge, just a disk component with a bar and an additional point-source in the center.

\subsection{Photometry of Clusters}

For detection of point sources in the HST images we used SExtractor [9] on the $I$ band image which was first un-sharp masked to highlight sources in the complex background of the ring. We used a similar strategy for SSC detections in [10]. Photometry was conducted on the original aligned broad-band images using fixed small apertures of 2 pixel $(0.1 \mathrm{arcsec})$ radius, with a sky-annulus from 2.5 to 4 pixel radii which corresponds to about $17 \mathrm{pc}$ physical size. Aperture corrections were adopted from [11] with effects of the re-sampling, and a larger PSF, on the $H$ band image determined using isolated point sources. All photometry was transformed to the Vegasystem [11]. The final photometric catalog at this point consists of 193 cluster candidates. We note that individual blue supergiants may still just be seen as individual sources at this limit, but they would not be numerous enough to make any statistical significance $[4,12]$ in our results which range to $M_{V} \approx-13.5 \mathrm{mag}$. The $\mathrm{H} \alpha$ image was treated separately. We obtained the $\mathrm{EW}(\mathrm{H} \alpha)$ values per YMC candidate as well as an empirical nebular emission correction to the $V$ and $H$ band magnitudes. These corrections can brighten the YMC when there is more emission in the sky annulus than in the target aperture. Typical differences between the corrected and uncorrected magnitudes are $\sim 0.1 \mathrm{mag}$ in $V$, while $\Delta$ mag values in the 0.2 to 0.6 range are found for some 20 sources in the strongest $\mathrm{H} \alpha$ emission areas.

We selected sources with photometric errors $<0.5$ mag in $V, I$ bands from the catalog of 193 sources produced above resulting in 159 cluster candidates. We checked their FWHMs, the great majority are clustered around the expected PSF size of $\sim 1.8$ pixels, the median being 1.9. We did not set a formal limit in $H$ mag error since we wanted an optically selected candidate list. Marginal NIR detections are thus included, but the $H$ band role of these cases is diminished due to inverse weighting by error. Results were checked not to change using the 129 sources resulting from setting $H$ error to $<0.5 \mathrm{mag}$, or by selecting stricter error cut-offs in all bands for that matter.

\section{Ages and masses of the YMCs}

It is well known that using only 3 broad band filters for mass/age modelling fails to break degeneracies between age, metallicity and extinction [13]. Especially ages between $\sim 10$ and $\sim 100$ Myr would be impossible to differentiate only with three band data. Constraining both metallicity and extinction with the SALT spectra at the ring locations helps, and most importantly we made use of $\mathrm{H} \alpha$ data. $V, I$ and $H$ band Starburst99 model ([14]; Padova tracks, Kroupa IMF, using $\mathrm{Z}=0.008$ ) magnitudes, which do not include nebular emission (though nebular continuum is

\footnotetext{
${ }^{4}$ NASA/IPAC Extragalactic Database
} 
included), were used as well as $\mathrm{EW}(\mathrm{H} \alpha)$ values. The $\chi^{2}$ minimisation fitting to these four points used the formal uncertainties from the photometry. $A_{V}$ was left as a free parameter, but restricted to range \pm 0.5 mag around 0.8 mag suggested by the spectra. The average of the output was $A_{V} \approx 0.9$. The masses were calculated based on the average of $V$ and $I$ luminosities after best-fit ages and extinctions were determined. The $H$ band luminosity was not used for mass determination since it is more susceptible to stochastic effects due to red supergiants $[15,5]$.

The clusters in NGC 2328 are young, ranging from a few Myr up to 30 Myr. Figure 2 summarises the luminosity function (LF), the mass function (MF), and also the age distribution of the detected sources. There is no evidence of any older population. Two key results are the apparent turnover of the MF (Fig. 2d), and an azimuthal age gradient along the ring (Fig. 3). We checked that the general characteristics of the mass and age distributions remain the same under a wide variety of modelling choices, including the input models themselves, making the main results robust.
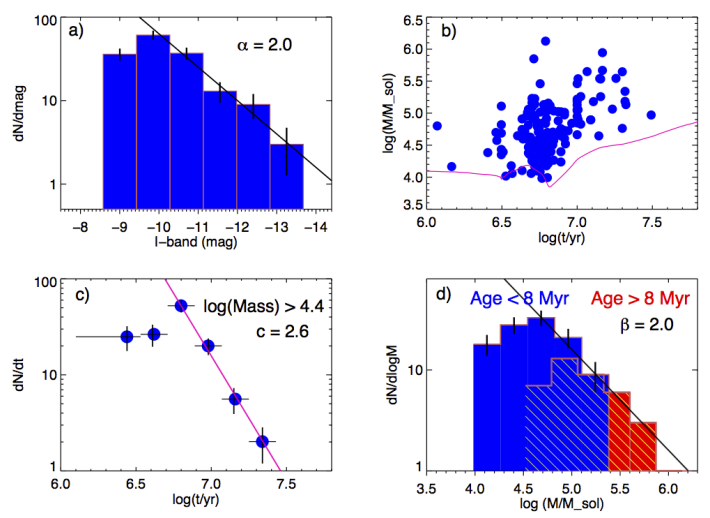

Figure 2: (a) The $I$ band LF of the YMCs with a canonical $\alpha=2$ power-law slope. (b) The mass vs. age of all the YMCs. The expected mass of a cluster at the $90 \%$ completeness limit, assuming $A_{V}=0.8 \mathrm{mag}$, is shown as the magenta curve. (c) The age distribution of the clusters above the indicated mass cutoff, with a power-law distribution of the oldest bins also shown. (d) The MF of YMCs below and above the age of 8 Myr are shown as the two histograms. A curve corresponding to a power-law slope of $\beta=2$ is overplotted.

We note that some fraction of the cluster candidates might be cluster complexes or blends of several SSCs. The surface density of YMCs in the ring area is about $600 \mathrm{kpc}^{-2}$, approaching confusion limits. However, with the small aperture sizes used, $r \approx 9 \mathrm{pc}$, crowding effects are not expected to significantly bias the statistical results [10]. The reasonably small apertures also help in mitigating filter-dependent resolution effects [16].

\section{The turnover of the mass function}

Figure 2a shows the luminosity function of all our YMCs fitted with $\Phi(L) d L \propto L^{-\alpha} d L$. The slope has a very robust $\alpha \sim 2$ until the 90\% completeness limit in agreement with "canonical" slopes for nearby star forming galaxies. The masses vs. age of the clusters is then shown in panel $\mathrm{b}$, with the mass limit vs. age corresponding to the photometric completeness indicated. The age distribution of the YMCs (panel c) at a complete mass range $\log \left(M_{\odot}\right)>4.4$, when fit with $d N / d \log (t) \sim t^{-c}$, gives $c \sim-2.6$ for $>5 \mathrm{Myr}$. This distribution is a complex function of 
cluster fading, dissolution, initial formation power-laws, and detection limits [17, 18, 19], and it is not obvious whether an analysis in the narrow age range we find is appropriate. Nevertheless, we note that the very steep slope is indicative of significant dissolution of clusters (dropping $\mathrm{H} \alpha$ and deriving older ages still results in $c \sim 1.5$ ).

The mass function is the most interesting distribution. The bright side of the MF in Fig. $2 d$ shows the typical $\beta \sim 2$ in a $d N / d M \propto M^{-\beta}$ fit. Though $\beta$ varies by at least \pm 0.5 depending on various binning and modelling choices, the MF turnover is very robust, whether all or subpopulations of ages are plotted. The blue histogram shows the youngest $<8$ Myr population, for example. Given the $90 \%$ completeness limit corresponds to $\sim 10^{4} M_{\odot}$ at the relevant ages the turnover cannot be due to photometric incompleteness only. A natural explanation of this behaviour is a rapid destruction of clusters at low masses - assuming that clusters are born in a power-law distribution, as widely accepted from observations of GMCs and open clusters in our own Milky Way, and more massive young clusters in the Local Group and beyond.

The result from this analysis thus is that while the more massive clusters appear to have typical YMC characteristics, the less massive cluster population in NGC 2328 has had to disappear in a very short timescale of 5 to $30 \mathrm{Myr}$ given the photometric depth of our images. This would also indicate that such clusters do not typically survive to become old globular clusters - YMCs thrown out of the densest birth regions by, e.g. interactions might have a better chance of survival [3].

\section{Age gradient}

Figure 3 plots the azimuth (compare to Fig. 1) of each YMC against its age, revealing a possible non-random distribution. It appears there is a minimum at $A Z \sim 60^{\circ}$ to $90^{\circ}$, and another one somewhere in-between $\mathrm{AZ}=230^{\circ}$ and $270^{\circ}$. While systematic trends over the whole system are somewhat speculative given the uncertainties, for example the youngest and oldest $45^{\circ}$ bins are just over $1 \sigma$ away from the mean, a gradient can be defined better in a section of the ring: The red curve in Fig. 3 shows a fit to the ages of individual cluster points over most of the North-East arm and the resulting slope has an uncertainty $3 \sigma$ away from a flat line. A similar, albeit much weaker gradient, could conceivably be fit on the SW arm as well, in between $\mathrm{Az} \sim 180^{\circ}$ to $300^{\circ}$. The PA of the identified bar is marked and it appears that the youngest SSCs are located some $\sim 50^{\circ}$ upstream from the points where the bar crosses the rotating ring, while the oldest areas correspond approximately to the ends of the two nuclear spiral arms. [20] find, from a sample of several nuclear rings, that the youngest HII regions tend to be correlated with contact points of the ring and bar induced flows they define to be perpendicular to the bar PA. Dust lanes normally reveal locations of these contact points, and indeed some are seen close to the locations of the youngest $\mathrm{YMCs}$ at $\mathrm{AZ} \sim 80^{\circ}$ and $260^{\circ}$ (Fig. 1) also corresponding to regions of highest nebular emission. The difference between the youngest clusters and the bar PA is similar to what [21] find for HII regions in the nuclear ring of IC 4933. Though it is difficult to exactly define the contact points in our case the mere presence of an age gradient is consistent with a dynamically (e.g. bar) induced cluster formation in the ring, a "pearls-on-a-string" scenario [22, 23] rather than localised gravitational instabilities resulting in a more random pattern (a "pop-corn model"). Also, any systematics in the age distribution indicate that multiple rotations, the rotational period of material at the ring radius is $\sim 30 \mathrm{Myr}$, have not washed out the signal confirming the generally young ages of clusters in NGC 2328. Interestingly, 
according to models of [23], merely finding an age gradient suggests the ring would have to be in a state of lower than average star formation rate (SFR). Investigating how this fits with the time scales of cluster formation, and in particular cluster formation efficiency (CFE) which can be estimated using the newly derived MF and the SFR of the ring from our $\mathrm{H} \alpha$ image, will be done in our follow-up work on the SF characteristics of the whole galaxy. We merely note here that CFE in the ring seems to be very high, much higher than in disks of ordinary SF galaxies. We find no evidence for an azimuthally systematic extinction effect, which could potentially explain the apparent age gradient, along the ring from the colour-maps, SSC age/mass/extinction fits, nor from the SALT spectra.

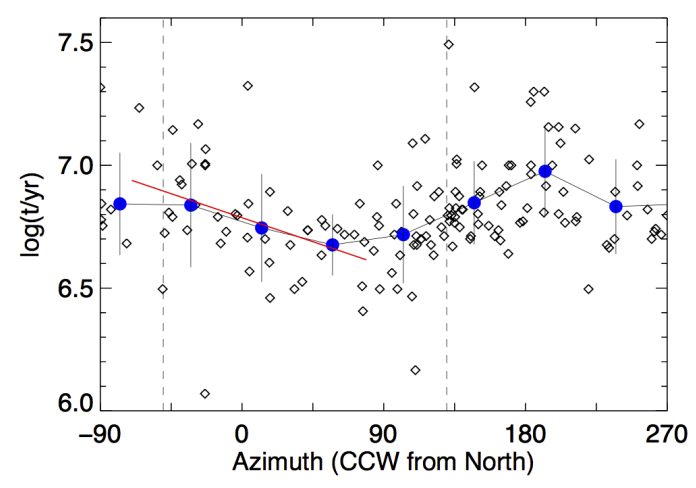

Figure 3: The ages of YMCs vs. AZ, counter-clockwise along the rotational direction. The blue points depict the averages in $45^{\circ}$ bins with the standard deviation of ages in each bin plotted as the uncertainty. The red curve shows a linear fit to the individual points in the $A Z=-70^{\circ}$ to $80^{\circ}$ range. The dashed vertical lines indicate the bar positions. See Fig. 1

\section{SUMMARY}

Massive star clusters appear to be forming very efficiently in a low-luminosity early type galaxy NGC 2328. The clusters in a nuclear spiral/ring are young, possibly all $<30 \mathrm{Myr}$. The young massive clusters show an azimuthal age gradient, at least over large sections of the ring, suggesting bar driven gas flow at a not-too-high rate, a pearls-on-a-string cluster formation scenario. We observe a robust turn-over in the mass function of the clusters. Assuming clusters are born with a power-law distribution, this turn-over indicates extremely rapid disruption. If such cases are found to be typical, it would indicate the YMCs created in tight SF rings do not survive to become old globular clusters. What in the environment is destroying them? While the high density and shear are likely to be involved we are investigating the physical properties of the galaxy itself in more detail to better understand the formation and dissolution mechanisms.

\section{References}

[1] Portegies Zwart, S. F., McMillan S. L. W. \& Gieles M., Young massive star clusters, 2010, ARAA, 48,431 
[2] Buta, R., Treuthardt, P. M., Byrd, G. G., Crocker, D. A., Circumnuclear Star Formation in the Early-Type Resonance Ring Barred Spiral Galaxy NGC 1326, 2000, AJ, 120, 1289

[3] Kruijssen, J. M. D. 2014, Classical and Quantum Gravity for a focus issue on Galactic Centres, Eds. Clifford Will and Pau Amaro-Seoane, IOP [arXiv:1407.2953]

[4] Maoz, D., Barth, A. J., Ho, L. C., Sternberg, A., Filippenko, A. V., An Ultraviolet through Infrared Look at Star Formation and Super Star Clusters in Two Circumnuclear Starburst Rings, 2001, AJ, 121, 3048

[5] de Grijs, R., Anders, P., Zackrisson, E., Östlin, G., The NGC 5253 star cluster system âĂŞ I. Standard modelling and infrared-excess sources, 2013, MNRAS, 431, 2917

[6] Krist, J., \& Hook, R. 2004, The Tiny Tim User's Guide (Version 6.3)

[7] de Vaucouleurs, G., de Vaucouleurs, A., Corwin, H. G., Jr., et al. 1991, Third Reference Catalogue of Bright Galaxies. Volume I: Explanations and references. Volume II: Data for galaxies between $0^{h}$ and $12^{h}$. Volume III: Data for galaxies between $12^{h}$ and $24^{h}$., New York, NY (USA), 2091

[8] Peng, C. Y., Ho, L. C., Impey, C. D., \& Rix, H.-W., Detailed structural decomposition of galaxy images, 2002, AJ, 124, 266

[9] Bertin, E. \& Arnouts, S., SExtractor: Software for source extraction, 1996, A\&ASS, 117, 393

[10] Randriamanakoto, Z., Väisänen, P., Ryder, S., Kankare, E., Kotilainen, J., \& Mattila, S., The K-band luminosity functions of super star clusters in luminous infrared galaxies, their slopes and the effects of blending, 2013, MNRAS, 431, 554

[11] Dolphin, A. E., A Revised Characterisation of the WFPC2 CTE Loss, 2009, PASP, 121, 655

[12] Chandar, R., Whitmore, B. C., Kim, H. et al., The Luminosity, Mass, and Age Distributions of Compact Star Clusters in M83 Based on Hubble Space Telescope/Wide Field Camera 3 Observations, 2010a, ApJ, 719, 966

[13] Anders, P., Bissantz, N., Fritze-v. Alvensleben, U., de Grijs, R., Analysing observed star cluster SEDs with evolutionary synthesis models: systematic uncertainties, 2004, MNARS, 347, 196

[14] Leitherer, C., Schaerer, D., Goldader, J. D., et al., Starburst99: synthesis models for galaxies with active star formation, 1999, ApJS 123, 3

[15] Gazak, J. Z., Bastian, N., Kudritzki, R.-P., Adamo, A., Davies, B., Plez, B., Urbaneja, M. A., Age dating stellar populations in the near infrared: an absolute age indicator from the presencelabsence of red supergiants, 2013, MNRAS, 430, 35

[16] Bastian, N., Adamo, A. Schirmer, M., et al., The effect of spatial resolution on optical and near-IR studies of stellar clusters: implications for the origin of the red excess, 2014, MNRAS, 4443829

[17] Boutloukos, S. G. \& Lamers, H. J. G. L. M., Star cluster formation and disruption time-scales - I. An empirical determination of the disruption time of star clusters in four galaxies, 2003, MNRAS, 338, 717

[18] Gieles, M., Lamers, H. J. G. L. M., Portegies Zwart, S. F., On the interpretation of the age distribution of star clusters in the Small Magellanic Cloud, 2007, ApJ, 668, 268

[19] Chandar, R., Whitmore, B. C, Fall, M., A comparison of methods for determining the age distribution of star clusters: application to the Large Magellanic Cloud 2010b, ApJ, 713, 1343

[20] Mazzuca, L. M., Knapen, J. H., Veilleux, S., Regan, M. W., A connection between star formation in nuclear rings and their host galaxies, 2008, ApJS, 174, 337 
[21] Ryder, S., Illingworth, S. M., Sharp, R. G., Farage, C. L., The nuclear ring in the barred spiral galaxy IC 4933, 2010, PASA, 27, 56

[22] Böker, T., Falcón-Barroso, J., Schinnerer, E., Knapen, J. H., Ryder, S., A SINFONI view of galaxy centers: morphology and kinematics of five nuclear star formation-rings, 2008, AJ, 135, 479

[23] Seo, W.-Y. \& Kim, W.-T., Star formation in nuclear rings of barred galaxies, 2013, ApJ, 769, 100 